\title{
Not Toeing the Number Line for Simple Arithmetic: Two Large-N Conceptual Replications of Mathieu et al. (Cognition, 2016, Experiment 1)
}

\author{
Jamie I. D. Campbell ${ }^{1}$ (D) , Yalin Chen ${ }^{1}$, Maham Azhar ${ }^{1}$ \\ [1] Department of Psychology, University of Saskatchewan, Saskatoon, Canada.
}

Journal of Numerical Cognition, 2021, Vol. 7(3), 248-258, https://doi.org/10.5964/jnc.6051

Received: 2019-09-05 • Accepted: 2020-02-13 • Published (VoR): 2021-11-30

Handling Editors: Mojtaba Soltanlou, University of Surrey, Guildford, UK; Krzysztof Cipora, Loughborough University, Loughborough, UK

Corresponding Author: Jamie I. D. Campbell, Department of Psychology, University of Saskatchewan, 9 Campus Drive, Saskatoon, SK, Canada, S7N 5A5. Phone 306-914-2691, Fax 306-966-1959. E-mail: jamie.campbell@usask.ca

Related: This article is part of the JNC Special Issue "Direct and Conceptual Replication in Numerical Cognition”, Guest Editors: Mojtaba Soltanlou \& Krzysztof Cipora, Journal of Numerical Cognition, 7(3), https://doi.org/10.5964/jnc.v7i3

Supplementary Materials: Data, Materials [see Index of Supplementary Materials]

\begin{abstract}
We conducted two conceptual replications of Experiment 1 in Mathieu, Gourjon, Couderc, Thevenot, and Prado (2016, https://doi.org/ 10.1016/j.cognition.2015.10.002). They tested a sample of 34 French adults on mixed-operation blocks of single-digit addition (4 +3$)$ and subtraction $(4-3)$ with the three problem elements $(\mathrm{O} 1,+/-, \mathrm{O} 2)$ presented sequentially. Addition was $34 \mathrm{~ms}$ faster if $\mathrm{O} 2$ appeared $300 \mathrm{~ms}$ after the operation sign and displaced $5^{\circ}$ to the right of central fixation, whereas subtraction was $19 \mathrm{~ms}$ faster when $\mathrm{O} 2 \mathrm{was}$ displaced to the left. Replication Experiment $1(n=74$ recruited at the University of Saskatchewan) used the same non-zero addition and subtraction problems and trial event sequence as Mathieu et al., but participants completed blocks of pure addition and pure subtraction followed by the mixed-operation condition used by Mathieu et al. Addition RT showed a 32 ms advantage with O2 shifted rightward relative to leftward but only in mixed-operation blocks. There was no effect of O2 position on subtraction RT. Experiment 2 ( $n=74$ ) was the same except mixed-operation blocks occurred before the pure-operation blocks. There was an overall 13 ms advantage with $\mathrm{O} 2$ shifted right relative to leftward but no interaction with operation or with mixture (i.e., pure vs mixed operations). Nonetheless, the rightward RT advantage was statistically significant for both addition and subtraction only in mixed-operation blocks. Taken together with the robust effects of mixture in Experiment 1, the results suggest that $\mathrm{O} 2$ position effects in this paradigm might reflect task specific demands associated with mixed operations.
\end{abstract}

\section{Keywords}

replication, simple addition and subtraction, spatial attention

Cognitive links between the representation and processing of number and space are indicated by numerous behavioral, neuropsychological and brain imaging studies (Fias \& Bonato, 2018; Fischer \& Shaki, 2018; Knops, 2018; Shaki, Pinhas, \& Fischer, 2018; Walsh, 2003). Dehaene, Bossini, and Giraux (1993) first demonstrated a relationship between numerical and spatial cognition using a parity-judgment task in which participants decided whether a single-digit number was odd or even by making a left- or right-side keyboard response. A response time (RT) advantage occurred for left-side responses to small numbers (i.e, 1, 2, 3, 4) and right-side responses to large numbers (i.e., 6, 7, 8, 9) compared to reverse mapping of response side and number size. Dehaene et al. named this the spatial-numerical association of response codes (SNARC) effect and proposed that it reflects a mental number line (MNL) representation of numerical magnitude 
that is spatially organized from left (smaller numbers) to right (large numbers). Since then, many experiments using a wide range of paradigms have confirmed a link between number magnitude and space (Casarotti, Michielin, Zorzi, \& Umiltà, 2007; Fischer, Castel, Dodd, \& Pratt, 2003; Fischer \& Shaki, 2018; Shaki, Fischer, \& Petrusic, 2009; Viarouge, Hubbard, \& Dehaene, 2014; see also Fias \& Van Dijck, 2016).

Beyond judgements about individual numbers, directional effects have also been observed in connection with performance of addition and subtraction (e.g., Hartmann, Mast, \& Fischer, 2016; Li, Liu, Li, Dong, Huang, \& Chen, 2018; Liu, Cai, Verguts, \& Chen, 2017; Masson \& Pesenti, 2014; Masson, Pesenti, Coyette, Andres, \& Dormal, 2017; Mathieu, Epinat-Duclos, Sigovan, et al., 2018; Mathieu, Gourjon, Couderc, Thevenot, \& Prado, 2016; McCrink, Dehaene, \& Dehaene-Lambertz, 2007; Pinhas \& Fischer, 2008; Pinhas, Shaki, \& Fischer, 2015; Pinheiro-Chagas, Dotan, Piazza, \& Dehaene, 2017). Of particular interest here is the paradigm developed by Mathieu et al. (2016, Experiment 1). This is a well-known paper with more than 60 citations in just a few years but there are several reasons to pursue a replication. First, this experiment was among the first (see also Wiemers, Bekkering, \& Lindemann, 2014) to demonstrate evidence of a visuospatial attention shift acting on arithmetic performance itself, as opposed to an effect of arithmetic operation on efficiency of visuospatial attention (e.g., Masson \& Pesenti, 2014). Consequently, it is important to affirm its replicability. Second, most studies of an association between space and arithmetic have used multi-digit problems $(78+6,78-6)$, whereas Mathieu et al. tested single-digit items (e.g., $8+6,8$ - 6). Solving multi-digit problems, however, includes solving one or more single-digit component problems; therefore, the mechanism of spatial associations for single-digit problems would also contribute to corresponding effects in multi-digit problems. This accentuates the importance of pursuing spatial effects for single-digit problems. Third, as explained further on, some of the Mathieu et al. results were not statistically compelling,

Mathieu et al. (2016, Experiment 1) analyzed the results of 34 students from the University of Lyon, France, on elementary addition and subtraction problems in random mixed-operation blocks. In their Experiment 1 paradigm, single-digit addition and subtraction problems (e.g., $5+4,5-4)$ were displayed with the first operand (O1), the operator (+ or -$)$, and the second operand (O2) presented sequentially. The spatial position of $\mathrm{O} 2$ was displaced either $5^{\circ}$ to the left or to the right of the central fixation point where the first two elements appeared. The inter-stimulus interval (ISI) between the operator and $\mathrm{O} 2$ was 150,300 or $450 \mathrm{~ms}$. Mathieu et al. (2016) refer to these intervals as stimulus onset asynchronies (SOAs), but their Figure 1 correctly depicted ISIs of 150, 300 or $450 \mathrm{~ms}$ following offset of the $150 \mathrm{~ms}$ operator. $^{1}$ Therefore, the SOAs between operator onset and O2 in their Experiment 1 were 300, 450 or 600 ms, whereas the ISI between the operator and O2 offset was 150, 300 or $450 \mathrm{~ms}$. Although this is a potentially important discrepancy for comparing the time course of spatial effects in the literature, it has little bearing on the present issue of replication.

With the $300 \mathrm{~ms}$ ISI there was strong statistical evidence reported (p. 233) that O2 position had different effects on RT for addition and subtraction, $F(1,33)=11.16, p=.002, M S E=.006, \eta_{\mathrm{p}}^{2}=.25, \mathrm{BF}_{10}=24.28 .^{2}$ Specifically, addition was $34 \mathrm{~ms}$ faster on average with $\mathrm{O} 2$ displaced rightward relative to leftward, $t(33)=2.67, p=.012, S E=12.7, \eta^{2}=.18$, $\mathrm{BF}_{10}=4.77$, whereas subtraction was $19 \mathrm{~ms}$ faster with $\mathrm{O} 2$ displaced leftward relative to rightward, $t(33)=-2.10, p=.04$, $S E=9.0, \eta^{2}=.12$, but $\mathrm{BF}_{10}=1.45$. So, there was little evidence of an O2-leftward advantage for subtraction at the 300 ms ISI. At the $150 \mathrm{~ms}$ and $450 \mathrm{~ms}$ ISIs there was also little statistical evidence based on BF values we calculated that the position of $\mathrm{O} 2$ affected RT for either addition or subtraction. The authors concluded (p. 234) that "these results demonstrate that solving single-digit addition and subtraction problems is associated with on-line horizontal shifts of attention. This is consistent with the idea that these problems activate procedures that may involve shifts to the right or left of a MNL". Other features of the results were reported to substantiate this claim. A subset of 15 participants in Experiment 1, tested on the advice of a reviewer, were also presented with addition and subtraction problems involving

1) Jérôme Prado confirmed (25/11/2019) that Figure 1 in Mathieu et al. (2016) correctly depicted the trial procedure.

2) The Bayes Factor (BF) values reported in this paper were calculated using MorePower 6.0.4 (Campbell \& Thompson, 2012). The program implements the Bayesian Information Criterion (BIC) as proposed by Masson (2011; see also Jarosz \& Wiley, 2014; Nathoo \& Masson, 2016; Wagenmakers, 2007), which approximates the unit-information prior as a default objective Bayes prior probability (Wagenmakers, 2007). $\mathrm{BF}_{01}$ denotes the odds ratio of the null $\left(\mathrm{H}_{0}\right)$ over the alternative hypothesis $\left(\mathrm{H}_{1}\right)$ and $\mathrm{BF}_{10}$ is the odds ratio of $\mathrm{H}_{1}$ over $\mathrm{H}_{0}$. A conventional interpretation is that $\mathrm{BF}$ greater than 10 provides relatively strong evidence for the hypothesis, $\mathrm{BF}$ values of 3 to 10 provide moderate evidence, whereas $\mathrm{BF}$ less than 3 provides little evidence one way or the other for $\mathrm{H}_{0}$ or $\mathrm{H}_{1}$ (e.g., Wetzels, van Ravenzwaaij, \& Wagenmakers, 2015). 
zero (e.g., $3+0,3-0$ ), which did not present effects of O2 position at the $300 \mathrm{~ms}$ ISI, $25 \mathrm{~ms}, t(14)=0.22, p=.83$, $S E=113.6, \eta^{2}=.003, \mathrm{BF}_{01}=3.77$; but no test of effects of $\mathrm{O} 2$ position on zero vs. non-zero problems was reported. The reported result fits with the attentional shift theory because zero-problems "should not require any shift along the MNL" (p. 231). Furthermore, Experiment $2(n=22)$ tested blocks of mixed single-digit addition and multiplication problems based on the same number pairs as Experiment 1 and using only the $300 \mathrm{~ms}$ ISI between the operator $(+$ or $\mathrm{x})$ and $\mathrm{O} 2$. As in Experiment 1, addition presented an RT advantage with $\mathrm{O} 2$ displaced rightward compared to leftward, $36 \mathrm{~ms}, t(21)=3.01, p=.007, S E=11.96, \eta^{2}=.30, \mathrm{BF}_{10}=11.02$, whereas multiplication did not, $-12 \mathrm{~ms}, t(21)=0.79$, $p=.44, S E=15.19, \eta^{2}=.03, \mathrm{BF}_{01}=3.40$. The results for addition replicated Experiment 1 and the null effect of $\mathrm{O} 2$ position for multiplication was predicted based on previous evidence that multiplication facts are retrieved directly from long-term memory (e.g., Campbell \& Xue, 2001) and would not involve the MNL. Mathieu et al. concluded that there is RT facilitation for non-zero single-digit subtraction and addition problems when the spatial position of the second operand in a sequential presentation of problem elements is congruent with the operation-dependent scan direction on a $\mathrm{MNL}$, running rightward up the number line for single-digit addition and leftward down the number line for single-digit subtraction.

\section{The Replication Experiments}

We conducted conceptual replications of Mathieu et al. (2016, Experiment 1). While the evidence was strong that addition benefitted from a rightward $\mathrm{O} 2$ shift compared to a leftward shift, the evidence for subtraction benefitting from a leftward shift was weak $\left(\mathrm{BF}_{10}=1.45\right)$ despite $p<.05$. It would be worthwhile to provide additional evidence about this finding. Furthermore, rather than the effect of $\mathrm{O} 2$ position reflecting an intrinsic spatial-attentional component of simple addition and subtraction, instead the Mathieu et al. results might reflect demand characteristics of their experimental paradigm. After presentation of $\mathrm{O} 1 \mathrm{for} 500 \mathrm{~ms}$ the operation to be performed was not determined until the operator appeared $500 \mathrm{~ms}$ after O1 offset. The operation sign (+ or -) was displayed very briefly (150 ms) followed by a delay of $150 \mathrm{~ms}$ up to $450 \mathrm{~ms}$ before the second operand (O2) appeared. Following encoding of O1, how is the operator information encoded to prepare for problem processing once $\mathrm{O} 2$ appears? The requirement to remember which operation to perform might induce a strategy to encode the operation sign as a spatial instruction about direction relative to $\mathrm{O} 1$ (e.g., if + then answer higher than O1; if - then answer lower than O1), akin to the more-or-less heuristic identified by Shaki et al. (2018). Use of a task-dependant spatial strategy to encode operation could be sensitive to the congruency of the direction planned (i.e., higher or lower) and shifts in the spatial location of $\mathrm{O} 2$. In this view, encoding $\mathrm{O} 2$ for addition is faster with shifts to the right and encoding of $\mathrm{O} 2$ for subtraction faster with shifts to the left. To eliminate this possible task-specific explanation our conceptual replications included pure addition and pure subtraction conditions along with the mixed-operation condition employed by Mathieu et al. We tested the pure-operation conditions first because practicing the mixed-operation condition first could induce a strategic procedure that is carried over to the pure-operation conditions.

\section{Experiment 1}

\section{Method}

\section{Participants}

The power is .9 for a sample of 72 or more to detect an operation-specific $\mathrm{O} 2$ position effect about half the size $\left(\eta_{\mathrm{p}}^{2}=.13\right)$ observed by Mathieu et al. Experiment 1 for the $300 \mathrm{~ms}$ ISI condition, $F(1,33)=11.16, \eta_{\mathrm{p}}^{2}=.25$ for the main effect of operation; p. 233. We tested 74 and replaced two participants with high error rates especially on large addition problems (sum > 10). The final sample included 45 women and 29 men, aged 17 to 30 years, $(M=20.8, S D=3.3)$, with 71 right-handed. They were recruited at the University of Saskatchewan and received course credit or $\$ 7.50$ CAD. Sixty reported English as their first language for arithmetic, three Mandarin, two reported Chinese, two reported Spanish and one each reported Afrikaans, Arabic, Bengali, Korean, Portuguese, Tagalog and Urdu. 


\section{Apparatus}

A Microsoft Windows-based computer used E-Prime 2.0 software (Schneider, Eschman, \& Zuccolotto, 2012) to display stimuli on a 15 inch CRT monitor. A chin rest was centered in front of the monitor at a distance of about $40 \mathrm{~cm}$. A microphone contected via the E-prime 2.0 response box provided the stop signal to measure verbal RT.

\section{Stimuli and Design}

The addition and subtraction stimuli were the same as Mathieu et al. (2016, Experiment 1). Small problems utilized the number pairs 21313241424351525354 and the larger problems were based on pairs 657576858687959697 98. These pairs were used for both the addition and subtraction problems (e.g., the digit pair 54 yields $5+4$ and $5-4$ ). Stimuli appeared in black, Courier New 36-point font with a white background. This was inadvertently different from Mathieu et al. (2016, Experiment 1), which displayed white characters on a black background.

Participants received three conditions: pure addition, pure subtraction, and mixed-operation addition and subtraction problems. Each condition comprised four trial blocks and within each block each problem appeared once with problem order randomized. For the first block of each condition, a random half of the problems were assigned to the O2-left condition where $\mathrm{O} 2$ appeared $5^{\circ}$ to the left of the central fixation point while the other half were assigned to the $\mathrm{O} 2$-right condition with $\mathrm{O} 2$ appearing $5^{\circ}$ to the right of fixation. The position of $\mathrm{O} 2$ for each problem then alternated across blocks resulting in each problem being tested twice with each $\mathrm{O} 2$ position. The order of the pure addition and pure subtraction conditions was counterbalanced across participants and the pure conditions always occurred prior to the mixed-operations condition. This avoided the possibilty that practice with mixed operations could have carry-over effects on potential spatial processes engaged in the pure-operation conditions.

\section{Procedure}

The 30-minute experimental session took place in a quiet room with an experimenter present. Instructions emphasized both speed and accuracy. There was no performance feedback given to the participant. For each trial participants held the microphone in their preferred hand with their chin on the chin rest. The sequence of events on each trial (see Figure 1) was the same as the $300 \mathrm{~ms}$ ISI condition in Mathieu et al. (2016, Experiment 1).

Figure 1

Trial Event Sequence in the Replication Studies of Mathieu et al. (2016) Experiment 1

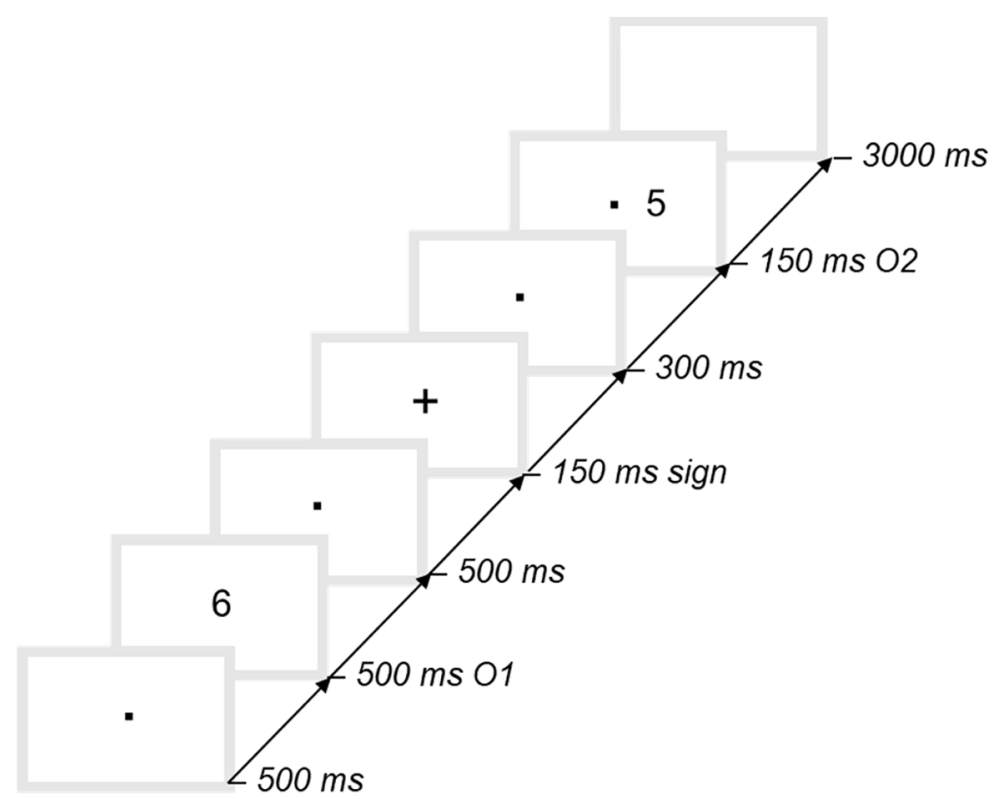


The trial started with a central fixation dot for $500 \mathrm{~ms}$. The first operand (O1) then appeared at the fixation point for $500 \mathrm{~ms}$ followed by the fixation dot for $500 \mathrm{~ms}$. Then the operator (+ or -) was displayed for $150 \mathrm{~ms}$ and replaced by the fixation dot for $300 \mathrm{~ms}$. The second operand (O2) appeared to the left or right of the fixation dot for $150 \mathrm{~ms}$ followed by a blank screen for up to $3000 \mathrm{~ms}$, which was the maximum time allowed for a response. Response timing began with the presentation of $\mathrm{O} 2$ and stopped when the participant spoke their answer into the microphone. During the $3150 \mathrm{~ms}$ response window, when a response was detected the screen switched immediately to a blank response recording screen. This allowed the experimenter to flag RTs that were spoiled because the microphone failed to detect the onset of the spoken response. Once the participant's response was recorded the fixation dot for the next trial appeared.

\section{Results}

\section{Analysis}

We analyzed the data using an Operation (addition, subtraction) $\times \mathrm{O} 2$ Position (left vs. right) $\times$ Mixture (pure vs. mixed-operation blocks) repeated-measures design. Mathieu et al. (2016) included problem size (small vs. large) as a factor but found that problem size had no important effects with respect to the O2 position manipulation. Therefore, we omitted it in our reported analysis to simplify the design. Mathieu et al. also performed a log transform on RTs prior to analysis. Our results were essentially the same with or without a log transform on RT and we report the latter. For completeness, however, the Supplementary Materials include a JASP file (JASP Team, 2019) containing the data and analyses with RTs log transformed and coded by problem size. ${ }^{3}$ Mathieu et al. reported one-tailed $t$-tests for analyses of spatial effects, although a one-tailed assmption is questionable given that this experiment was the first trial of their new paradigm. Nonetheless, in their Experiment 1 these tests were nominally significant using a two-tailed criterion. We report $p$-values for two-tailed tests throughout.

\section{Response Time}

A total of 1124 RTs (4.7\%) were excluded from the analysis because they exceeded the $3150 \mathrm{~ms}$ second response deadline, were marked as spoiled by the experimenter, or were more than $4 S D$ from a participant's mean RT in each Operation $\times$ O2 Position $\times$ Mixture cell. Table 1 presents mean RT and $S E$ for correct answers as a function of operation, mixture, and O2 position. On average, addition was slower $(988 \mathrm{~ms})$ than subtraction $(838 \mathrm{~ms}), F(1,73)=88.41, p<.001, M S E=37544$, $\eta_{\mathrm{p}}^{2}=.55, \mathrm{BF}_{10}>10000$. There was weak evidence of an interaction between operation and mixture, $F(1,73)=4.52, p=.04$, $M S E=7332, \eta_{\mathrm{p}}^{2}=.06$, but $\mathrm{BF}_{10}=1.07$, reflecting slower subtraction in mixed-operation blocks relative to pure-operation blocks (854 vs $822 \mathrm{~ms}$ ), $t(73)=2.64, p=.01, S E=11.87, \eta^{2}=.09, \mathrm{BF}_{10}=3.40$, whereas mean addition RT did not differ between pure-operation $(987 \mathrm{~ms})$ and mixed-operations blocks $(989 \mathrm{~ms}), t(73)=.11, p=.92, S E=13.19, \eta^{2}=.0002$, $\mathrm{BF}_{01}=8.55$. We might expect mixed-operation blocks to be slower than pure-operation blocks for both operations owing to task switching or mixing effects (e.g., Campbell \& Arbuthnott, 2010). Mixed-operation blocks always followed pure-operation blocks, however, and the same problems were tested in the pure-operation and mixed-operation blocks. Consequently, mixed-operation RT costs would be masked by facilitative effects of practice following pure-operation blocks.

There was a Mixture $\times \mathrm{O} 2$ Position interaction, $F(1,73)=7.94, p=.006, M S E=1841, \eta_{\mathrm{p}}^{2}=.10, \mathrm{BF}_{10}=5.30$, owing to an overall $18 \mathrm{~ms}$ RT advantage with $\mathrm{O} 2$ appearing to the right of fixation in mixed operation blocks, $t(73)=3.01, p=.004$, $S E=5.92, \eta^{2}=.11, \mathrm{BF}_{10}=8.69$, but no effect of $\mathrm{O} 2$ position in pure-operation blocks [mean RT difference of $-2 \mathrm{~ms}, t(73)=$ -0.36, $\left.p=.72, S E=5.87, \eta^{2}=.002, \mathrm{BF}_{01}=8.07\right]$. There also was weak evidence for the three-way interaction, $F(1,73)=$ 5.97, $p=.02, M S E=2267, \eta_{\mathrm{p}}^{2}=.08, \mathrm{BF}_{10}=2.13$. As Table 1 shows, in mixed-operation blocks the mean RT for addition was $32 \mathrm{~ms}$ faster with $\mathrm{O} 2$ appearing to the right than to the left of fixation, $t(73)=3.39, p=.001, S E=9.31, \eta^{2}=.14$, $\mathrm{BF}_{10}=25.62$, but $\mathrm{O} 2$ position had no effect on addition $\mathrm{RT}$ in pure-operation blocks, $-7.5 \mathrm{~ms}, t(73)=-0.88, p=.38, \mathrm{SE}=$

\footnotetext{
3) Log transformation is a common remedy to normalize positively skewed RT distributions, but factors such as problem size in arithmetic studies can distribute RTs from some experimental cells disproportionately into the upper tail of the experiment-wide distribution of RTs (Campbell \& Penner-Wilger, 2006). Upper tail RTs are affected most by log normalization; consequently, log transformations can mask genuine effects on RT or produce misleading artefacts. Both the benefits and potential costs of RT transformations need to be considered (Whelan, 2008).
} 
$8.58, \eta^{2}=.01, \mathrm{BF}_{01}=5.85$. A separate analysis that included only addition trials confirmed a difference in the effect of $\mathrm{O} 2$ position between the mixed- and pure-operation conditions, $F(1,73)=11.26, p=.001, M S E=2499, \eta_{\mathrm{p}}^{2}=.13, \mathrm{BF}_{10}=23.46$. $^{4}$

Table 1

Performance in Experiment 1

\begin{tabular}{|c|c|c|c|c|c|c|}
\hline \multirow[b]{2}{*}{ Mixture } & \multicolumn{3}{|c|}{ Add } & \multicolumn{3}{|c|}{ Subtract } \\
\hline & O2 Left & O2 Right & $L-R$ & O2 Left & O2 Right & L - R \\
\hline \multicolumn{7}{|l|}{ RT } \\
\hline Pure & $983(29)$ & 991 (31) & $-7.5(8.6)$ & $824(22)$ & $821(22)$ & $3.3(6.8$ \\
\hline Mixed & $1004(34)$ & $972(33)$ & $31.5(9.3)$ & $856(26)$ & $852(26)$ & $4.1(7.1$ \\
\hline \multicolumn{7}{|l|}{ Errors } \\
\hline Pure & $4.3(0.8)$ & $4.6(0.7)$ & $-0.3(0.5)$ & $2.1(0.4)$ & $1.4(0.3)$ & $0.6(0.4$ \\
\hline Mixed & $4.7(0.6)$ & $5.0(0.7)$ & $-0.3(0.4)$ & $2.8(0.5)$ & $2.1(0.3)$ & $0.7(0.3$ \\
\hline
\end{tabular}

Note. Mean RT and mean percentage of errors (SE in brackets) in Experiment 1 by operation, O2 position and mixture. L $-\mathrm{R}=\mathrm{O} 2 \mathrm{Left}-\mathrm{O} 2 \mathrm{Right}$.

In contrast to addition, the results showed no effect on subtraction RT of the left vs. right position of O2 in either pure-operation, $3 \mathrm{~ms}, t(73)=.49, p=.62, S E=6.75, \eta^{2}=.003, \mathrm{BF}_{01}=7.61$, or mixed-operation blocks, $4 \mathrm{~ms}, t(73)=.57$, $p=.57, S E=7.13, \eta^{2}=.005, \mathrm{BF}_{01}=7.29$. Thus, the mixed-operation condition replicated the effects of the spatial position of $\mathrm{O} 2$ on addition RT observed by Mathieu et al. (2016), but we did not replicate an effect of O2 position on subtraction RT.

We used the MSE values generated in a Mixture $\times$ O2 Position ANOVA of the subtraction RT data to estimate expected variability in potential effects of $\mathrm{O} 2$ position. Using MorePower 6.0.4, we determined that the experiment had power of .9 for a main effect of $\mathrm{O} 2$ position of $17 \mathrm{~ms}$ or greater $(M S E=1958)$, and power of .98 to detect a Mixture $\times \mathrm{O} 2$ Position interaction effect as large as that observed in the addition data $(39 \mathrm{~ms}, M S E=1609)$. Thus, the experiment was virtually certain to have detected a comparable effect of $\mathrm{O} 2$ position in the subtraction data, had it existed.

\section{Percentage of Errors}

Mathieu et al. (2016, Experiment 1) did not present an analysis of errors. For completeness here, Table 1 includes mean percentage of errors (i.e., the rate of incorrect arithmetic answers) for each Operation $\times$ O2 Position $\times$ Mixture cell. A factorial analysis is not warranted because 250 of the 592 cells in the data set (i.e., $2 \times 2 \times 2 \times 74$ participants) had a value of 0 (i.e., were at the measurement floor). This potentially inflates the Type I error rate owing to artificially low variances.

\section{Discussion}

Experiment 1 replicated Mathieu et al. (2016) with respect to faster addition with O2 rightward than leftward of fixation, but subtraction did not demonstrate the reverse pattern reported by Mathieu et al., although the experiment had ample power to detect an effect for subtraction. Additionally, the effect of $\mathrm{O} 2$ position in addition occurred only in the mixed-operations condition and not in pure addition blocks. This raises the possibility that effects of $\mathrm{O} 2$ position in this paradigm arise from demand characteristics of the mixed operations combined with a very brief $150 \mathrm{~ms}$ display of the operator to cue operation. A potentially important factor, however, distinguishing Experiment 1 here and the Mathieu et al. Experiment 1 is that the mixed-operation condition used by Mathieu et al. was preceded here by pure blocks of

4) As observed by Mathieu et al. (2016), the RT advantage for addition in mixed-operation blocks with $\mathrm{O} 2$ appearing rightward of fixation compared to O2 leftward of fixation was observed both for numerically smaller (sum $\leq 10)$ problems, $22 \mathrm{~ms}, t(73)=2.68, p=.009, S E=8.23, \eta^{2}=.09, \mathrm{BF} 10=3.77$, and larger $($ sum $>10)$ problems, $40 \mathrm{~ms}, t(73)=2.59, p=.01, S E=15.35, \eta^{2}=.08, \mathrm{BF}_{10}=2.97$. 
addition and subtraction. In Experiment 2, we reversed the situation with the mixed-operation condition preceding the pure-operation conditions.

\section{Experiment 2}

\section{Method}

Seventy-four people who had not participated in Experiment 1 (45 women and 29 men, aged 18 to 54 years, $M=27.7$, $S D=7.6,67$ right-handed) were recruited as in Experiment 1. Forty-seven reported English as their first language for arithmetic, nine Persian, six Mandarin, four reported Spanish, three Vietnamese, two Farsi, and one each reported Bangla, Bengali, Chinese, Portuguese, Telugu and Urdu.

The apparatus, stimuli, design and procedure were the same as Experiment 1 except that the mixed-operation condition was presented before the pure addition and pure subtraction tasks. The order of the pure-operation tasks was counterbalanced across participants.

\section{Results}

\section{Response Time}

A total of 1318 RTs (5.6\%) were excluded from the analysis by the same criteria as Experiment 1 . The RT data from Experiment 2 were analyzed by operation, $\mathrm{O} 2$ position and mixture as in Experiment 1 . The Supplementary Materials include a JASP file containing the data and analyses with RTs log transformed and coded by problem size to match Mathieu et al. (2016, Experiment 1). Table 2 presents the mean correct RT and SE for each cell. On average, addition was slower $(942 \mathrm{~ms})$ than subtraction $(810 \mathrm{~ms}), F(1,73)=96.60, p<.001, M S E=26897, \eta_{\mathrm{p}}^{2}=.57, \mathrm{BF}_{10}>10000$, and mixed-operation blocks were slower on average (898 ms) than pure operation blocks (854 ms), $F(1,73)=25.88, p<.001$, $M S E=11540, \eta_{\mathrm{p}}^{2}=.26, \mathrm{BF}_{10}>10000$. As in Experiment 1 , there was some evidence of an interaction between operation and mixture, $F(1,73)=6.64, p=.01, M S E=3146, \eta_{\mathrm{p}}^{2}=.08$, but $\mathrm{BF}_{10}=2.91$, although the form of the interaction was different, probably reflecting the reversed order of the mixed and pure operation conditions: Subtraction was $33 \mathrm{~ms}$ slower in mixed-operation blocks relative to pure blocks (826 vs. $793 \mathrm{~ms}$ ), $t(73)=4.22, p<.001, S E=7.83, \eta^{2}=.20$, $\mathrm{BF}_{10}=372.91$, whereas mean addition RT was $57 \mathrm{~ms}$ slower in mixed compared to pure blocks (971 vs. $914 \mathrm{~ms}$ ), $t(73)=4.85, p<.00001, S E=11.71, \eta^{2}=.24, \mathrm{BF}_{10}=3587.37$. This pattern likely reflects, at least in part, $\mathrm{RT}$ gains from practice of problems in the mixed-operation condition transferring to the same problems tested again subsequently in the pure-operation conditions, with greater RT gains for the relatively more-difficult addition problems.

Table 2

Performance in Experiment 2

\begin{tabular}{|c|c|c|c|c|c|c|}
\hline \multirow[b]{2}{*}{ Mixture } & \multicolumn{3}{|c|}{ Add } & \multicolumn{3}{|c|}{ Subtract } \\
\hline & O2 Left & O2 Right & $\mathbf{L}-\mathbf{R}$ & O2 Left & O2 Right & $\mathbf{L}-\mathbf{R}$ \\
\hline \multicolumn{7}{|l|}{ RT } \\
\hline Pure & 918 (27) & $909(26)$ & $9.1(8.3)$ & 799 (17) & $788(16)$ & $10.6(5.9)$ \\
\hline Mixed & 979 (29) & $962(28)$ & $16.9(8.3)$ & $835(18)$ & $818(18)$ & $16.5(8.0)$ \\
\hline \multicolumn{7}{|l|}{ Errors } \\
\hline Pure & $3.9(0.8)$ & $3.8(0.7)$ & $0.1(0.5)$ & $1.8(0.3)$ & $2.1(0.4)$ & $-0.3(0.4)$ \\
\hline Mixed & $5.8(0.9)$ & $6.5(1.0)$ & $-0.7(0.6)$ & $3.6(0.5)$ & $2.6(0.6)$ & $1.0(0.4)$ \\
\hline
\end{tabular}

Note. Mean RT and mean percentage of errors (SE in brackets) in Experiment 2 by operation, O2 position and mixture. L $-\mathrm{R}=\mathrm{O} 2 \mathrm{Left}-\mathrm{O} 2 \mathrm{Right}$.

There was also a main effect of $\mathrm{O} 2$ position because mean RT with O2 displaced rightward (869 ms) was slightly faster overall than with $\mathrm{O} 2$ leftward $(883 \mathrm{~ms}), F(1,73)=9.68, p<.003, M S E=2698, \eta_{\mathrm{p}}^{2}=.12, \mathrm{BF}_{10}=11.62$. Inspection of Table 
2 might suggest that this effect was larger in mixed-operation blocks, $17 \mathrm{~ms}, t(73)=2.66, p=.01, S E=6.27, \eta^{2}=.09$, $\mathrm{BF}_{10}=3.57$, than pure blocks, $10 \mathrm{~ms}, t(73)=1.85, p=.07, S E=5.33, \eta^{2}=.04, \mathrm{BF}_{10}=1.57$, but the test of the interaction disconfirmed this possibility, $F(1,73)=0.743, p=.39, M S E=2316, \eta_{\mathrm{p}}^{2}=.01, \mathrm{BF}_{01}=5.91$. More importantly, there was good evidence against an Operation $\times \mathrm{O} 2$ Position interaction, $F(1,73)=0.007, p=.94, M S E=1503, \eta_{\mathrm{p}}^{2}<.001, \mathrm{BF}_{01}=8.57$, as well as against the presence of a three-way interaction, $F(1,73)=0.016, p=.90, M S E=2182, \eta_{\mathrm{p}}^{2}<.001, \mathrm{BF}_{01}=8.53 .^{5}$

\section{Percentage of Errors}

The overall mean rate of incorrect arithmetic answers was 3.8\% of trials. Table 2 includes mean percentage of errors for each Operation $\times$ O2 Position $\times$ Mixture cell. Again, a factorial analysis is not warranted given that 260 of the 592 cells in the data set contained a value of 0 , the measurement floor.

\section{Discussion}

With respect to $\mathrm{O} 2$ position effects, Experiment 2 replicated only the rightward position RT advantage for addition observed in Experiment 1, but in this experiment subtraction also showed some evidence of a similar rightward advantage, and $\mathrm{O} 2$ position did not interact with arithmetic operation as in Experiment 1. Both addition and subtraction showed a significant effect of $\mathrm{O} 2$ position only in the mixed-operation condition, but the test of the interaction with pure vs. mixed operations strongly supported a null effect rather than the interaction observed in Experiment 1.

\section{General Discussion}

We replicated Mathieu et al. (2016, Experiment 1) with respect to a RT advantage for addition when the position of $\mathrm{O} 2$ was shifted rightward relative to $\mathrm{O} 2$ in a position left of fixation. We found no evidence, however, that subtraction RTs were faster with $\mathrm{O} 2$ shifted leftward; in fact in Experiment 2 there was some evidence that subtraction was faster with $\mathrm{O} 2$ shifted to the right. Nonetheless, there is evidence from a variety of experimental paradigms that subtraction is associated with a leftward direction or location (e.g., Blini, Pitteri, \& Zorzi, 2019; Li et al., 2018; Liu, Cai, Verguts, \& Chen, 2017; Masson \& Pesenti, 2014; Zhu, You, Gan, \& Wang, 2019), but there are also other exceptions (e.g., Masson, Letesson, \& Pesenti, 2018). It may be, however, that the Mathieu et al. paradigm does not reliably engage this spatial association for subtraction. As mentioned previously, their own evidence for an effect for subtraction was very weak with a $\mathrm{BF}_{10}$ value of only 1.45 . We conclude that their paradigm using the $300 \mathrm{~ms}$ ISI before O2 is sensitive to a rightward spatial bias for addition but not sensitive to a generalized leftward spatial association for subtraction.

Furthermore, in Experiment 1 the RT gain for addition with O2 rightward occurred only in mixed-operation trial blocks and not in the pure-addition blocks; but we did not replicate an effect of mixed vs. pure-operation blocks in Experiment 2. Perhaps, with the mixed-operation condition first as in Experiment 2, participants acquired a problem-encoding procedure to accommodate the trial-by-trial unpredictability of the operation to be performed after O1 was presented. This task set could then persist into the subsequent pure-operation blocks, washing out mixture effects in Experiment 2 that were robust in Experiment 1.

The present results raise the possibility that spatial bias effects in the Mathieu et al. (2016) experiments were the product of the task-set procedures that participants develop to handle unpredictable intermixed arithmetic operations. Randomly mixing operations across trials is a common practice in research into spatial biases in arithmetic; but mixing may invite spatial strategies such as the more-or-less heuristic (Shaki et al., 2018) that produce spatial effects not necessarily intrinsic to performance of addition or subtraction generally but rather arise as artefacts of paradigm-specific task demands. This is consistent with evidence that spatial and numerical representations must be jointly involved to observe

\footnotetext{
5) Twelve of the 74 participants reported that their first language for arithmetic was Farsi, Persian or Urdu, which are languages with right-to-left reading direction. The SNARC effect appears to be sensitive to participants' habitual reading direction such that right-to-left readers have demonstrated a reverse SNARC effect (Dehaene et al., 1993; Shaki et al., 2009) suggesting a reverse mapping of number magnitude to space between right-to-left and left-to-right readers (see also Azhar, Chen, \& Campbell, 2020). To our knowledge effects of reading direction on number-space associations have not been investigated for simple arithmetic. We repeated the analyses for Experiment 2 excluding right-to-left readers and found the same pattern of results for RT with the remaining 62 participants. This analysis was included among the Supplementary Materials.
} 
effects suggesting a spatially organised MNL (Pinto, Pellegrino, Marson, Lasaponara, \& Doricchi, 2019). As mentioned by Mathieu et al. (p. 234), the plus sign (+) cues that the answer will be more than O1 whereas the minus sign (-) cues that it will be less than $\mathrm{O} 1$. We suggest that this spatial encoding of operation (i.e., go up or down relative to O1) may be particularly likely if the operator sign is presented very briefly in the context of mixed-operation trials. With this operation encoding strategy, the addition operator shifts attention rightward, facilitating encoding of O2 when it appears on the right relative to the left side. In this view, the effect is not intrinsic to the addition process per se, but instead resides in speeding up $\mathrm{O} 2$ encoding time. In theory, this spatial encoding effect would be reversed for subtraction, although our experiments did not find an advantage for subtraction with $\mathrm{O} 2$ displaced leftward. This result, combined with the O2-rightward RT advantage in Experiment 2 for both addition and subtraction, indicate that no single or simple mechanism can explain the present results. Nonetheless, the context sensitivity of spatial effects in the Mathieu et al. paradigm suggests that the task does not measure an automatic, functionally integrated spatial processing component for single-digit addition or subtraction.

Funding: This work was supported by a Discovery Grant awarded to Jamie Campbell by the Natural Sciences and Engineering Research Council of Canada.

Acknowledgments: The authors have no additional (i.e., non-financial) support to report.

Competing Interests: The authors report no conflicts or competing interests.

Ethics Approval: This research was approved on ethical grounds by the Behavioural Research Ethics Board of the University of Saskatchewan, Canada.

Data Availability: For this article, a dataset is freely available (Campbell, Chen, \& Azhar, 2020).

\section{Supplementary Materials}

The Supplementary Materials contain the data, materials, and code for this study (for access see Index of Supplementary Materials below).

\section{Index of Supplementary Materials}

Campbell, J. I. D., Chen, Y., \& Azhar, M. (2020). Supplementary materials to "Not toeing the number line for simple arithmetic: Two large$n$ conceptual replications of Mathieu et al. (Cognition, 2016, Experiment 1)" [Research data, materials, and code]. OSF.

https://osf.io/9b45h/

Journal of Numerical Cognition. (Ed.). (2021). Supplementary materials to "Not toeing the number line for simple arithmetic: Two large-n conceptual replications of Mathieu et al. (Cognition, 2016, Experiment 1)" [Open peer-review]. PsychOpen GOLD.

https://doi.org/10.23668/psycharchives.5221

\section{References}

Azhar, M., Chen, Y., \& Campbell, J. I. D. (2020). Reading direction and spatial effects in parity and arithmetic tasks. Psychological Research. Advance online publication. https://doi.org/10.1007/s00426-020-01397-y

Blini, E., Pitteri, M., \& Zorzi, M. (2019). Spatial grounding of symbolic arithmetic: An investigation with optokinetic stimulation. Psychological Research, 83(1), 64-83. https://doi.org/10.1007/s00426-018-1053-0

Campbell, J. I. D., \& Arbuthnott, K. A. (2010). Effects of mixing and cuing simple addition and multiplication. European fournal of Cognitive Psychology, 22, 422-442. https://doi.org/10.1080/09541440902903629

Campbell, J. I. D., \& Penner-Wilger, M. (2006). Calculation latency: The $\mu$ of memory and the $\tau$ of transformation. Memory \&

Cognition, 34, 217-226. https://doi.org/10.3758/BF03193400

Campbell, J. I. D., \& Thompson, V. A. (2012). MorePower 6.0 for ANOVA with relational confidence intervals and Bayesian analysis.

Behavior Research Methods, 44, 1255-1265. https://doi.org/10.3758/s13428-012-0186-0 
Campbell, J. I. D., \& Xue, Q. (2001). Cognitive arithmetic across cultures. Fournal of Experimental Psychology: General, 130, $299-315$. https://doi.org/10.1037/0096-3445.130.2.299

Casarotti, M., Michielin, M., Zorzi, M., \& Umiltà, C. (2007). Temporal order judgment reveals how number magnitude affects visuospatial attention. Cognition, 102(1), 101-117. https://doi.org/10.1016/j.cognition.2006.09.001

Dehaene, S., Bossini, S., \& Giraux, P. (1993). The mental representation of parity and number magnitude. fournal of Experimental Psychology: General, 122(3), 371-396. https://doi.org/10.1037/0096-3445.122.3.371

Fias, W., \& Bonato, M. (2018). Which space for numbers. In A. Henik \& W. Fias (Eds.), Heterogeneity of function in numerical cognition (pp. 233-242). https://doi.org/10.1016/B978-0-12-811529-9.00002-9

Fias, W., \& Van Dijck, J. (2016). The temporary nature of number-Space interactions. Canadian fournal of Experimental Psychology/ Revue canadienne de psychologie expérimentale, 70(1), 33-40. https://doi.org/10.1037/cep0000071

Fischer, M. H., Castel, A. D., Dodd, M. D., \& Pratt, J. (2003). Perceiving numbers causes spatial shifts of attention. Nature Neuroscience, 6(6), 555-556. https://doi.org/10.1038/nn1066

Fischer, M. H., \& Shaki, S. (2018). Number concepts: Abstract and embodied. Philosophical Transactions of the Royal Society B: Biological Sciences, 373(1752), Article 20170125. https://doi.org/10.1098/rstb.2017.0125

Hartmann, M., Mast, F. W., \& Fischer, M. H. (2016). Counting is a spatial process: Evidence from eye movements. Psychological Research, 80(3), 399-409. https://doi.org/10.1007/s00426-015-0722-5

Jarosz, A. F., \& Wiley, J. (2014). What are the odds? A practical guide to computing and reporting Bayes factors. fournal of Problem Solving, 7, Article 2. https://doi.org/10.7771/1932-6246.1167

JASP Team. (2019). JASP (Version v0.10.2) [Computer software]. Retrieved from https://jasp-stats.org

Knops, A. (2018). Neurocognitive evidence for spatial contributions to numerical cognition. In A. Henik \& W. Fias (Eds.), Heterogeneity of function in numerical cognition (pp. 211-232). https://doi.org/10.1016/B978-0-12-811529-9.00011-X

Li, M., Liu, D., Li, M., Dong, W., Huang, Y., \& Chen, Q. (2018). Addition and subtraction but not multiplication and division cause shifts of spatial attention. Frontiers in Human Neuroscience, 12, Article 183. https://doi.org/10.3389/fnhum.2018.00183

Liu, D., Cai, D., Verguts, T., \& Chen, Q. (2017). The time course of spatial attention shifts in elementary arithmetic. Scientific Reports, 7(1), Article 921. https://doi.org/10.1038/s41598-017-01037-3

Masson, M. E. J. (2011). A tutorial on a practical Bayesian alternative to null-hypothesis significance testing. Behavior Research Methods, 43, 679-690. https://doi.org/10.3758/s13428-010-0049-5

Masson, N., Letesson, C., \& Pesenti, M. (2018). Time course of overt attentional shifts in mental arithmetic: Evidence from gaze metrics. Quarterly fournal of Experimental Psychology, 71(4), 1009-1019. https://doi.org/10.1080/17470218.2017.1318931

Masson, N., \& Pesenti, M. (2014). Attentional bias induced by solving simple and complex addition and subtraction problems. Quarterly fournal of Experimental Psychology, 67(8), 1514-1526. https://doi.org/10.1080/17470218.2014.903985

Masson, N., Pesenti, M., Coyette, F., Andres, M., \& Dormal, V. (2017). Shifts of spatial attention underlie numerical comparison and mental arithmetic: Evidence from a patient with right unilateral neglect. Neuropsychology, 31(7), 822-833.

https://doi.org/10.1037/neu0000361

Masson, N., Pesenti, M., \& Dormal, V. (2017). Impact of optokinetic stimulation on mental arithmetic. Psychological Research, 81(4), 840-849. https://doi.org/10.1007/s00426-016-0784-z

Mathieu, R., Epinat-Duclos, J., Sigovan, M., Breton, A., Cheylus, A., Fayol, M., . . Prado, J. (2018). What's behind a “+” sign? Perceiving an arithmetic operator recruits brain circuits for spatial orienting. Cerebral Cortex, 28(5), 1673-1684. https://doi.org/10.1093/cercor/bhx064

Mathieu, R., Gourjon, A., Couderc, A., Thevenot, C., \& Prado, J. (2016). Running the number line: Rapid shifts of attention in singledigit arithmetic. Cognition, 146, 229-239. https://doi.org/10.1016/j.cognition.2015.10.002

McCrink, K., Dehaene, S., \& Dehaene-Lambertz, G. (2007). Moving along the number line: Operational momentum in nonsymbolic arithmetic. Perception \& Psychophysics, 69(8), 1324-1333. https://doi.org/10.3758/BF03192949

Nathoo, F. S., \& Masson, M. E. J. (2016). Bayesian alternatives to null-hypothesis significance testing for repeated-measures designs. fournal of Mathematical Psychology, 72, 144-157. https://doi.org/10.1016/j.jmp.2015.03.003

Pinhas, M., \& Fischer, M. H. (2008). Mental movements without magnitude? A study of spatial biases in symbolic arithmetic. Cognition, 109(3), 408-415. https://doi.org/10.1016/j.cognition.2008.09.003

Pinhas, M., Shaki, S., \& Fischer, M. H. (2015). Addition goes where the big numbers are: Evidence for a reversed operational momentum effect. Psychonomic Bulletin \& Review, 22(4), 993-1000. https://doi.org/10.3758/s13423-014-0786-z 
Pinheiro-Chagas, P., Dotan, D., Piazza, M., \& Dehaene, S. (2017). Finger tracking reveals the covert stages of mental arithmetic. Open Mind, 1(1), 30-41. https://doi.org/10.1162/OPMI_a_00003

Pinto, M., Pellegrino, M., Marson, F., Lasaponara, S., \& Doricchi, F. (2019). Reconstructing the origins of the space-number association: Spatial and number-magnitude codes must be used jointly to elicit spatially organised mental number lines. Cognition, 190, 143-156. https://doi.org/10.1016/j.cognition.2019.04.032

Schneider, W., Eschman, A., \& Zuccolotto, A. (2012). E-Prime user's guide. Pittsburgh, PA, USA: Psychology Software Tools, Inc. Shaki, S., Fischer, M. H., \& Petrusic, W. M. (2009). Reading habits for both words and numbers contribute to the SNARC effect. Psychonomic Bulletin \& Review, 16(2), 328-331. https://doi.org/10.3758/PBR.16.2.328

Shaki, S., Pinhas, M., \& Fischer, M. H. (2018). Heuristics and biases in mental arithmetic: Revisiting and reversing operational momentum. Thinking \& Reasoning, 24(2), 138-156. https://doi.org/10.1080/13546783.2017.1348987

Viarouge, A., Hubbard, E. M., \& Dehaene, S. (2014). The organization of spatial reference frames involved in the SNARC effect. Quarterly fournal of Experimental Psychology, 67, 1484-1499. https://doi.org/10.1080/17470218.2014.897358

Wagenmakers, E.-J. (2007). A practical solution to the pervasive problems of p values. Psychonomic Bulletin \& Review, 14, 779-804. https://doi.org/10.3758/BF03194105

Walsh, V. (2003). A theory of magnitude: Common cortical metrics of time, space and quantity. Trends in Cognitive Sciences, 7, 483-488. https://doi.org/10.1016/j.tics.2003.09.002

Wetzels, R., van Ravenzwaaij, D., \& Wagenmakers, E.-J. (2015). Bayesian Analysis. In R. L. Cautin \& S. O. Lilienfeld (Eds.), The Encyclopedia of Clinical Psychology (pp. 274-283). https://doi.org/10.1002/9781118625392.wbecp453

Wiemers, M., Bekkering, H., \& Lindemann, O. (2014). Spatial interferences in mental arithmetic: Evidence from the motion-arithmetic compatibility effect. Quarterly Journal of Experimental Psychology, 67, 1557-1570. https://doi.org/10.1080/17470218.2014.889180

Whelan, R. (2008). Effective analysis of reaction time data. The Psychological Record, 58, 475-482. https://doi.org/10.1007/BF03395630

Zhu, R., You, X., Gan, S., \& Wang, J. (2019). Spatial attentions shifts in addition and subtraction arithmetic: Evidence of eye movement. Perception, 48, 835-849. https://doi.org/10.1177/0301006619865156

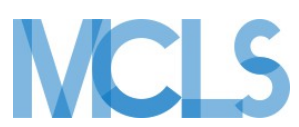

Journal of Numerical Cognition (JNC) is an official journal of the Mathematical Cognition and Learning Society (MCLS).
(P) leibniz-psychology.org

PsychOpen GOLD is a publishing service by Leibniz Institute for Psychology (ZPID), Germany. 